\title{
Evaluación del nivel de madurez en la gestión de proyectos de una empresa prestadora de servicios públicos
}

\author{
Evaluation of the level of maturity \\ in project management of a company \\ providing public services
}

Artículo de estudio de caso.

Fecha de recepción: 27/11/2020

Fecha de devolución: 24/04/2021

Fecha de aceptación: 05/05/2021

Fecha de publicación: 12/05/2021

\section{Carlos Alberto Gómez Cano}

Corporación Unificada Nacional de

Educación Superior - CUN

Florencia, Caquetá (Colombia) carlos_gomezca@cun.edu.co

\section{Verenice Sánchez Castillo \\ Universidad de la Amazonia \\ Florencia, Caquetá (Colombia) ve.sanchez@udla.edu.co}

Para citar este artículo:

Gómez-Cano, C. A. \& Sánchez-Castillo, V. (2021). Evaluación del nivel de madurez en la gestión de proyectos de una empresa prestadora de servicios públicos. Económicas CUC, 42(2), 133-144. DOI: https://doi.org/10.17981/ econcuc.42.2.2021.Org/7

\begin{abstract}
Resumen
Existe una relación directamente proporcional entre la efectividad en gestión de proyectos y el cumplimiento de los objetivos corporativos. Por tanto, consolidar un alto grado de madurez en la gestión de proyectos es un proceso imperioso para las organizaciones que basan su accionar en estos. El presente manuscrito muestra los resultados de la evaluación de la madurez en la gestión de proyectos de una empresa prestadora de servicios públicos en el departamento del Caquetá. La investigación presentó un enfoque mixto, pues recurrió a las encuestas y las entrevistas como instrumentos para la obtención de información. Los resultados fueron valorados bajo los postulados de Harold Kerzner, permitiendo evidenciar que la organización se encuentra en el primer nivel de madurez, lo cual no es sinónimo de caos total, pero sí de la necesidad de generar acciones desde la alta dirección para la consolidación de una cultura de proyectos, que permita, en el mediano plazo subsanar las deficiencias y potencializar las ventajas de la organización.

Palabras clave: Gestión de proyectos; madurez organizacional; cultura de proyectos
\end{abstract}

\begin{abstract}
There is a directly proportional relationship between project management effectiveness and the fulfillment of corporate objectives. Therefore, consolidating a high degree of maturity in project management is an imperative process for organizations that base their actions on these. This manuscript shows the results of the evaluation of the maturity in project management of a company providing public services in the department of Caquetá. The research presented a mixed approach, since it used surveys and interviews as instruments for obtaining information. The results were evaluated under the postulates of Harold Kerzner, allowing to show that the organization is at the first level of maturity, which is not synonymous with total chaos, but rather with the need to generate actions from senior management for the consolidation of a culture of projects that allows, in the medium term, to correct the deficiencies and enhance the advantages of the organization.

Keywords: Project management; organizational maturity; project culture
\end{abstract}




\section{INTRODUCCIÓN}

\section{La madurez en la gestión de proyectos, aproximaciones al concepto}

Si bien existen múltiples académicos que han contribuido a la definición del término "proyecto", dado su auge en las dinámicas académicas y empresariales (Gómez, Sánchez y Fajardo, 2018), el Project Management Institute-PMI (2017), a través de su Project Management Body of Knowledge (PMBOK), se consolidan como una autoridad en la materia, definiendo a estos como "un esfuerzo temporal que se lleva a cabo para crear un producto, servicio o resultado único. La naturaleza temporal de los proyectos implica que un proyecto tiene un principio y un final definidos" (p. 3), lo que permite deducir que existe una relación estrecha entre el éxito de las organizaciones y los proyectos, especialmente por el carácter flexible y dinámico de estos (Shek, 2013).

Consecuentemente con lo anterior, la gestión de proyectos es una disciplina que ha presentado grandes desarrollos en los últimos tiempos, y por ende, múltiples definiciones. Por ejemplo, Terrazas (2009) ha expresado:

\section{La gestión de proyectos apunta a la consideración de herramientas de tipo gerencial, las cuales deben ser enfocadas para que la empresa u organización sea capaz de desarro- llar un conjunto de habilidades tanto a nivel individual como de trabajo en equipo. El propósito de lograr esta actividad es planificar, organizar, dirigir y controlar los eventos asociados al proyecto, dentro de un escenario de tiempo, costo y calidad predetermina- $\operatorname{dos}($ p. 167).}

La anterior posición es compartida parcialmente por Moreno, Sánchez y Velosa (2018), quienes expresan que dentro de las actuales responsabilidades del gestor de proyecto está "generar valor a las compañías, no solamente se debe dedicar a controlar las variables que componen la triple restricción: alcance, tiempo y costos" (p. 21), es decir que, la gestión de proyecto tiene como propósito superior la generación de valor, accionar que desborda las acciones exclusivas de monitoreo y control, pues demanda "la aplicación de conocimientos, habilidades, herramientas y técnicas a las actividades del proyecto para cumplir con los requisitos del proyecto" (PMI, 2021). Sobre el particular, Gómez (2009) añade que "la gestión de proyectos implica un enfoque especial del trabajo, que no puede adoptarse sin considerar las maneras como la institución ha organizado sus recursos y acostumbra desarrollar el conjunto de sus actividades" (p. 38).

Ahora bien, en lo concerniente a la madurez en la gestión de proyectos, según el PMBOK, esta está ligado a la "capacidad de una organización para producir los resultados estratégicos deseados de un modo predecible, controlable y confiable" (PMI, 2017, p. 552), es decir que, a mayor grado de madurez en la organización, mayor certeza se poseerá sobre los resultados de los proyectos, convirtiéndolo en criterio de éxito organizacional (Arce \& López, 2010). En palabras de Parviz y Levin (2002), a mayor nivel de madurez, existe mayor calidad de entregables, disminución y control de erogaciones, equipos con altos índices de motivación y un equilibro entre costo- 
cronograma-calidad, lo que se traduce, evidentemente, en mejores resultados para la organización.

De lo anterior, se colige que la madurez en la gestión de proyectos esta ligada a mayores índices de productividad y competitividad (Nungo, Torres y Palacios, 2018), por tanto, para toda organización es deseable alcanzar y consolidar un alto nivel en dicha dimensión, y, para ello es indispensable la realización de constantes procesos de evaluación integral, que permitan develar las realidades de la empresa y de sus prácticas (Del Cioppo \& Bello, 2018). En línea con lo anterior, el objetivo principal de la esta investigación estuvo alrededor de evaluar el nivel de madurez de una empresa prestadora de servicios públicos en el departamento del Caquetá, para lo cual se tomó como referencia los planteamientos de Harold Kerzner, que se exponen a continuación.

\section{El modelo de Madurez de Harold Kerzner}

Si bien existen múltiples propuestas en el mundo académico para la medición de la madurez organizacional, uno de los modelos con mayor auge y reconocimiento, según indica Teherán y Torres (2019), es el propuesto por Harold Kerzner en su obra "Using the Project Management Maturity Model. Strategic Planning for Project Management", el cual luego de su publicación en el 2005, se ha convertido en un referente para este tipo de procesos. Tal y como se detalla en la Figura 1, se consideran cinco (05) niveles de madurez, iniciando en lenguaje común (básico) y terminando en mejoramiento continuo (óptimo), el detalle de cada uno de estos escaños se describe en la Tabla 1.

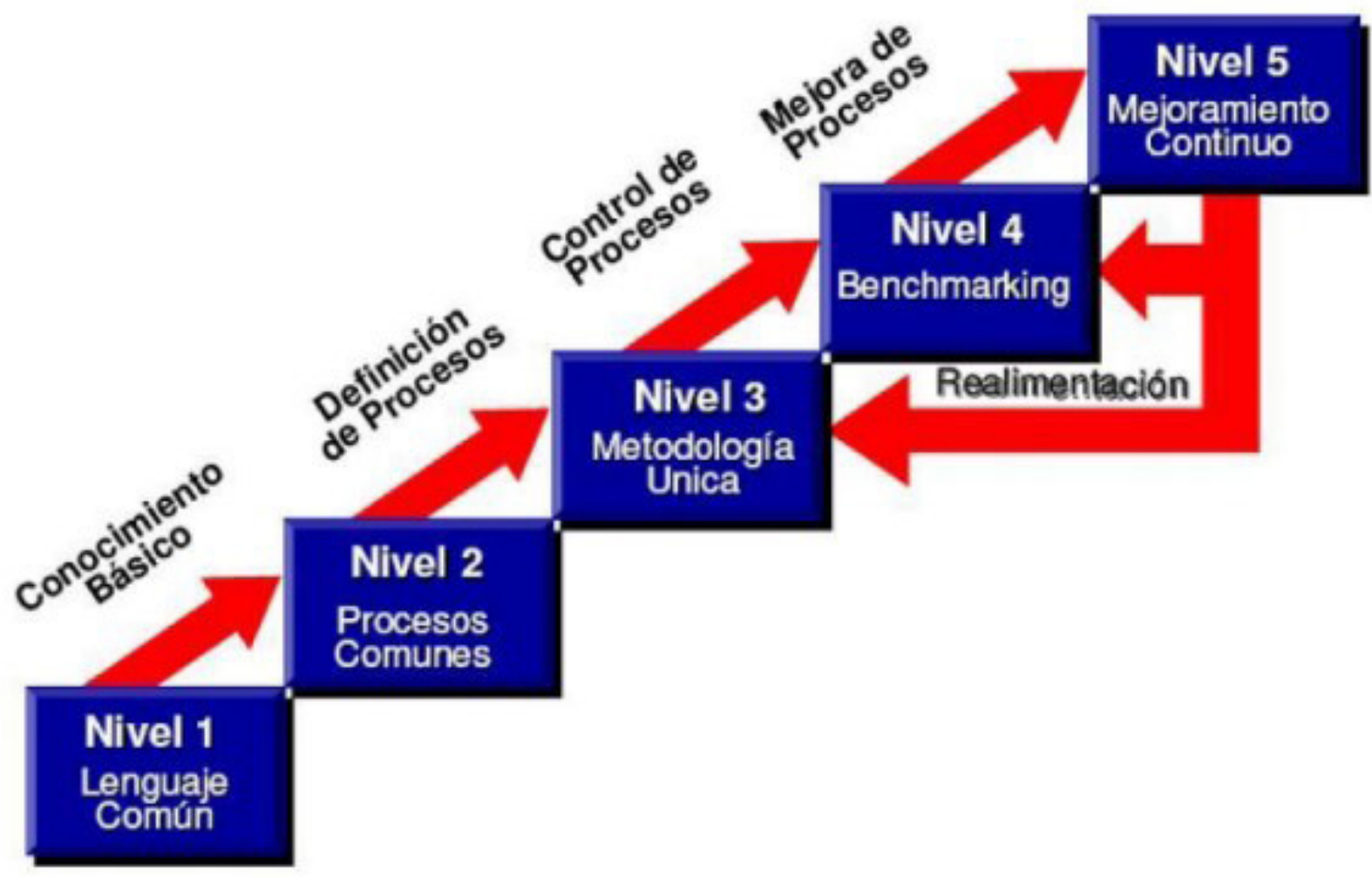

Figura 1. Escalas de madurez propuesta por Harold Kerzner.

Fuente: Kerzner (2005). 
TABLA 1.

Conceptualización de los niveles de madurez de Harold Kerzner.

\begin{tabular}{|c|c|}
\hline Nivel & Definición \\
\hline $\begin{array}{l}\text { Lenguaje } \\
\text { Común. }\end{array}$ & $\begin{array}{l}\text { La empresa reconoce tanto la importancia como la necesidad de conocer y emplear } \\
\text { técnicas propias de la gerencia de proyectos para poder alcanzar una base común } \\
\text { para la comunicación. }\end{array}$ \\
\hline $\begin{array}{l}\text { Procesos } \\
\text { Comunes. }\end{array}$ & $\begin{array}{l}\text { La necesidad de definir y desarrollar procesos comunes es reconocida por la } \\
\text { empresa en su propósito de alcanzar y repetir experiencias de éxito en la gestión de } \\
\text { proyectos.. }\end{array}$ \\
\hline $\begin{array}{l}\text { Metodología } \\
\text { Única. }\end{array}$ & $\begin{array}{l}\text { La organización es consciente del efecto que tiene la sinergia para hacer posible la } \\
\text { combinación o fusión eficaz de todas las metodologías corporativas en una única } \\
\text { metodología enfocada en la administración de proyectos. }\end{array}$ \\
\hline Benchmarking. & $\begin{array}{l}\text { La empresa identifica y actúa al reconocer la necesidad de perfeccionar sus procesos } \\
\text { para poder generar y mantener una ventaja competitiva significativa. Como } \\
\text { consecuencia de ese reconocimiento, establece a quien y sobre cuáles temas se } \\
\text { realizará el monitoreo necesario para realizar análisis comparativos que resulten } \\
\text { significativos para sus objetivos estratégicos. }\end{array}$ \\
\hline $\begin{array}{l}\text { Mejora } \\
\text { Continua. }\end{array}$ & $\begin{array}{l}\text { La organización valora la información que obtiene a través del benchmarking para } \\
\text { establecer si esta podría realmente mejorar su metodología previamente establecida. }\end{array}$ \\
\hline
\end{tabular}

Fuente: Elaboración propia a partir de Kerzner (citado por Triana, 2018, p. 46).

\section{Metodología}

Medir el nivel de madurez de una organización en la gestión de proyectos, según indica Wendler (2012), demanda un proceso de evaluación profundo y multidimensional, por lo que, consecuentemente, el presente ejercicio de investigación se desarrolló desde un enfoque mixto, el cual permite una lectura holística del contexto, facilitando la interpretación y análisis del fenómeno investigado (Gómez, Sánchez y Ramon, 2017).

Referente al alcance de la pesquisa es descriptivo, el cual apunta a especificar las propiedades, las características y los perfiles de personas, grupos, comunidades, procesos, objetos o cualquier otro fenómeno que se someta a un análisis" (Hernández-, Fernández y Baptista, 2014, p. 92), lo que, para el caso en particular, refiere a la caracterización en detalle de los procesos y reacciones organizacionales en torno a la gestión de proyecto en la empresa estudiada.

De esta manera, la investigación se desarrolló bajo las siguientes etapas:

Etapa I. Diagnóstico de las prácticas y efectividad en la gestión de proyectos. En desarrollo de esta fase, se aplicó la encuesta diseñada por Dora Alba Ariza Aguilera, la cual está compuesta por cincuenta (50) preguntas, que responden a tres subcapítulos, a saber: contexto e información general de la organización, donde se detallan elementos referentes a la estructura organizacional y las dinámicas de poder en torno a los proyectos; evaluación de las prácticas en torno a la gestión de proyectos, para lo cual se tomaron como referente las diez (10) áreas de conocimiento descritas en el PMBOK del PMI (2017); y, por último, medición de la efectividad en la gestión de proyectos, la cual se midió a través de indicadores como cumplimiento de expectativas, satisfacción del equipo vinculado al proyecto y logro de compromisos. Los resultados de la encuesta fueron procesados a través de la herramienta Excel. 
Etapa II. Identificación de las percepciones en torno a la dinámica de la gestión de proyectos por parte de los directivos y ejecutivos de la organización. Esta fase se construyó a partir de las entrevistas, las cuales tuvieron como preguntas orientadoras ¿Cómo se gestionan los proyectos al interior de la organización? ¿Cuáles han sido las dificultades/debilidades más sentidas en la gestión de proyectos? ¿Qué acciones considera usted se puede implementar para maximizar la efectividad en la gestión de proyectos?; los resultados fueron procesados a través del Software de análisis cualitativo Atlas ti (v. 6.0).

Etapa III. Definición del estatus de la organización en la gestión de proyectos, según el modelo de Harold Kerzner. En virtud de los hallazgos derivados de las etapas anteriores, se realizó una clasificación de la organización, según los criterios y patrones establecidos por el modelo de Harold Kerzner.

\section{RESUltados}

\section{Diagnóstico de las prácticas y efectividad en la gestión de proyectos}

En la Tabla 2 se consolidan los resultados de las quince (15) encuestas aplicadas a integrantes de la organización, quienes están vinculados directa o indirectamente con la gestión de proyectos. Los resultados, si se evalúan desde la tradición académica de aprobatorio para variables por encima de tres punto cero (3.0), dan cuenta de que la organización presenta limitaciones en el 50\% de las áreas de conocimiento para la gestión de proyectos, y, además, que solo el 30\% de estas están en un récord superior a tres punto cinco (3.5), lo que permite evidenciar grandes oportunidades de mejora para la empresa.

TABLA 2.

Resultados sobre prácticas en gestión de proyectos.

\begin{tabular}{ll}
\hline Área & \multicolumn{1}{c}{ Resultado } \\
\hline Alcance & 3.57 \\
Tiempo & 2.75 \\
Costo & 2.56 \\
Calidad & 3.36 \\
Riesgos & 1.08 \\
Adquisiciones & 3.68 \\
Stakeholders & 3.14 \\
Recursos Humanos & 2.73 \\
Comunicaciones & 2.85 \\
Cambio & 4.19 \\
\hline
\end{tabular}

Fuente: Elaboración propia a partir de las Encuestas.

Las áreas que presentan las valoraciones mas bajas son Riesgos (1.08), Costos (2.56) y Recursos Humanos (2.7), todas bastantes criticas e influyentes para el 
éxito de los proyectos en la organización, pues tienen repercusiones significativas sobre los resultados corporativos y el tejido empresarial (Sparano, 2011). Por el contrario, las áreas con mayores puntuaciones son Cambio (4.19), Adquisiciones (3.68) y Alcance (3.57), lo que daría cuenta de procesos estructurados, al menos desde la dimensión administrativa de la organización, siendo un indicador positivo para la consolidación de una cultura de proyectos en la organización (Gómez, 2019).

En la Tabla 3 se presentan los cómputos obtenidos sobre la efectividad de los proyectos, obteniendo el cumplimiento de expectativa la mayor calificación (3.48), lo que está relacionado con la naturaleza misma de la organización, pues para las empresas prestadoras de servicios públicos es parte de su propósito superior la satisfacción de sus usuarios. Por el contrario, la menor calificación está en torno al logro de compromisos (2.93), lo que refleja demoras e incumplimiento en la gestión de los proyectos (Gómez, 2019).

TABLA 3.

Resultados sobre efectividad en proyectos

\begin{tabular}{ll}
\hline Factor & \multicolumn{1}{c}{ Resultado } \\
\hline Cumplimiento de Expectativas & 3.48 \\
Satisfacción del Equipo de Proyecto & 3.36 \\
Logro de Compromisos & 2.93 \\
\hline
\end{tabular}

Fuente: Elaboración propia a partir de las Encuestas.

Identificación de las percepciones en torno a la dinámica de la gestión de proyectos por parte de los directivos y ejecutivos de la organización

En el presente apartado se presenta el resultado de las entrevistas, luego de haber sido transcritas, categorizadas y trabajadas a través del Software Atlas Ti, lo que dio vida a tres (03) categorías de análisis, así:

\section{Familia Económica}

Existen tres situaciones relacionadas, que describen la preocupación que en términos económicos tienen los entrevistados acerca de la gestión de los proyectos en la empresa. Primero mencionaron los altos presupuestos, y altos costos de inversión, que implica un proyecto relacionado con el sector en el que se encuentra matriculado la organización; esto en ocasiones limita la consecución de los recursos económicos requeridos para la realización total de una obra, razón por la cual muchas veces se hace por fases, pero algunas intervenciones solo quedan en la etapa inicial, luego se empiezan otros y otros y no se concluye. De manera contradictoria, cuando se obtienen unos recursos generosos para la inversión, esta no ha sido austera, generando despilfarro de los recursos, tal y como se detalla en la Figura 2. 


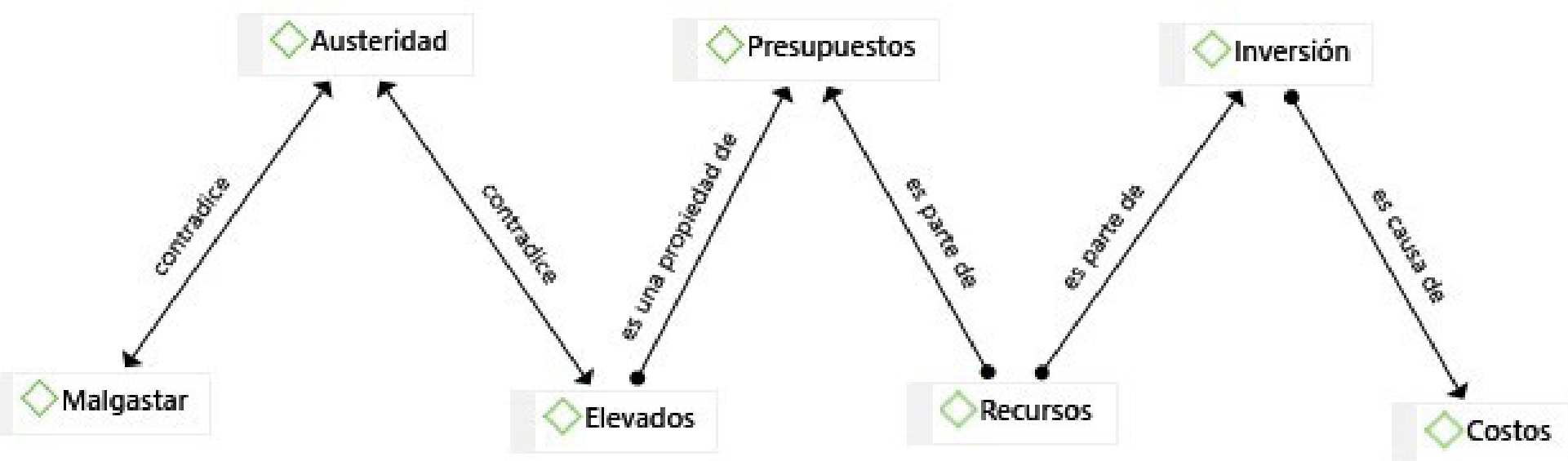

Figura 2. Familia Económica.

Fuente: Elaboración propia a partir de las entrevistas.

\section{Familia Estructura Organizacional}

Dada su naturaleza y trayectoria, la estructura organizacional, al menos desde la revisión documental, es expresada por la empresa como una de sus fortalezas para la gestión de sus proyectos. Sin embargo, de acuerdo con los relatos recolectados, esta dimensión lejos de ser una potencialidad o fortaleza de la empresa, es un asunto que se explicita por sus agentes como una gran debilidad, pues consideran que, si bien existe una estructura organizacional definida, esta es débil, con pocas claridades en su actuar y en la delegación de funciones, tampoco existe un patrón que permita la colaboración organizativa.

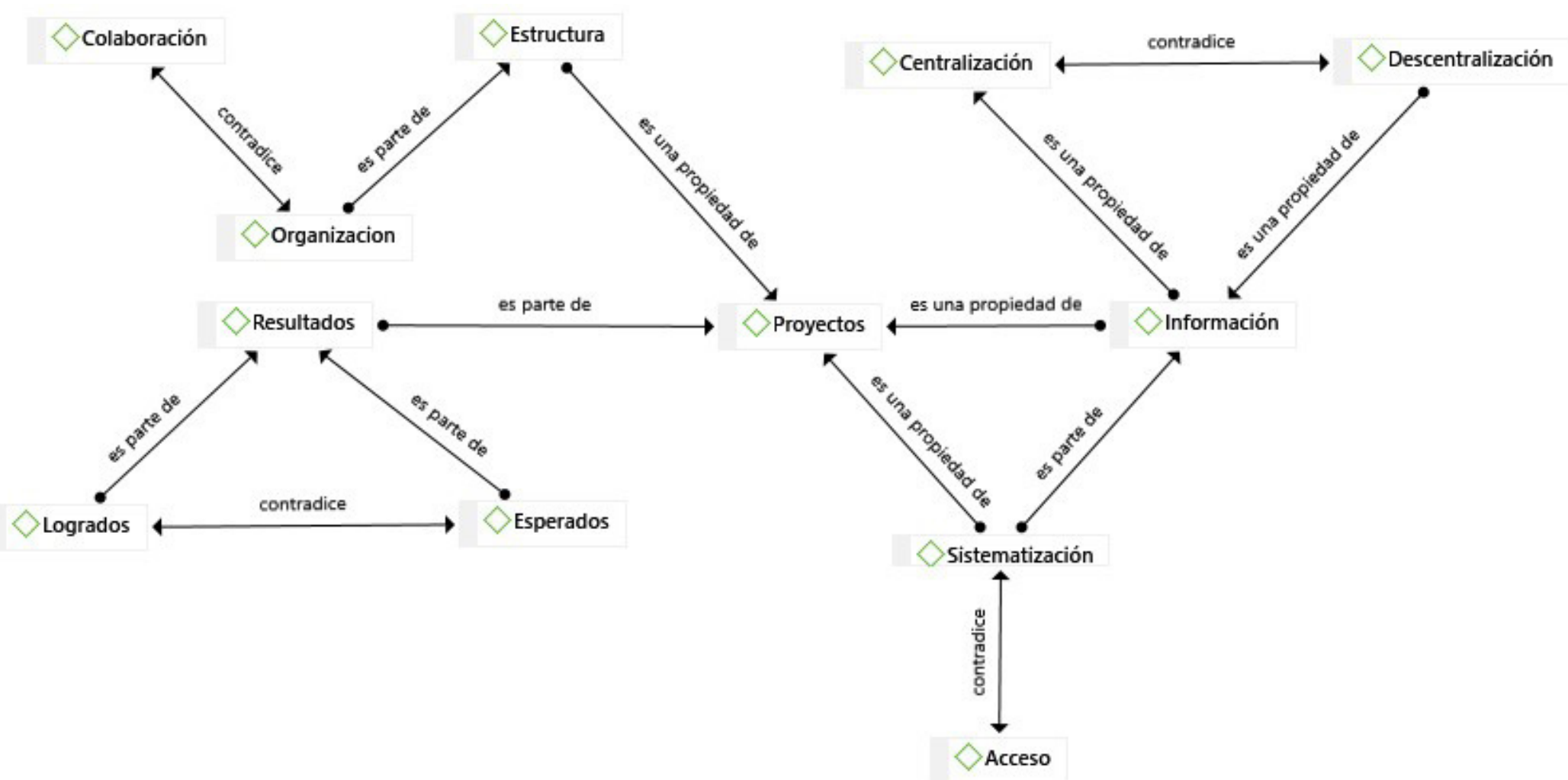

Figura 3. Familia Estructura Organizacional

Fuente: Elaboración propia a partir de las entrevistas. 
De igual forma ha permitido que algunos proyectos caigan en la temida centralización, en tanto en otras ocasiones, la flexibilidad ha hecho que la descentralización, ponga en riesgo los alcances en términos de presupuesto y resultados de los proyectos. Así mismo, aunque se reconoce que se hace una sistematización de la información rigurosa, permanente y detallada, dicha información, no se puede acceder de manera continua o inmediata en caso de una solicitud urgente. Finalmente, tal y como se evidencia en la Figura 3, mencionaron que, en la parte organizacional, no se ha trabajado con claridades en la determinación de las oportunidades y tampoco de los riesgos.

\section{Familia Planeación}

En este acápite los entrevistados dejaron ver en su relato categorías relacionadas, especialmente con el cronograma de actividades del proyecto, pues consideran que si bien en términos de la gestión de estos, la empresa realiza una planeación de las actividades a desarrollar, en unos tiempos y con un presupuesto destinado; este plan en reiteradas ocasiones, es incumplido, pues los directores y funcionaros de los proyectos llevan su propia dinámica y se salen de lo planeado inicialmente. Esta situación genera que el tiempo no alcance para lograr los objetivos trazados y que el cronograma diseñado sea no sea funcional, ni real. Esto se evidencia finalmente en el alcance del proyecto y los resultados obtenidos (Figura 4):

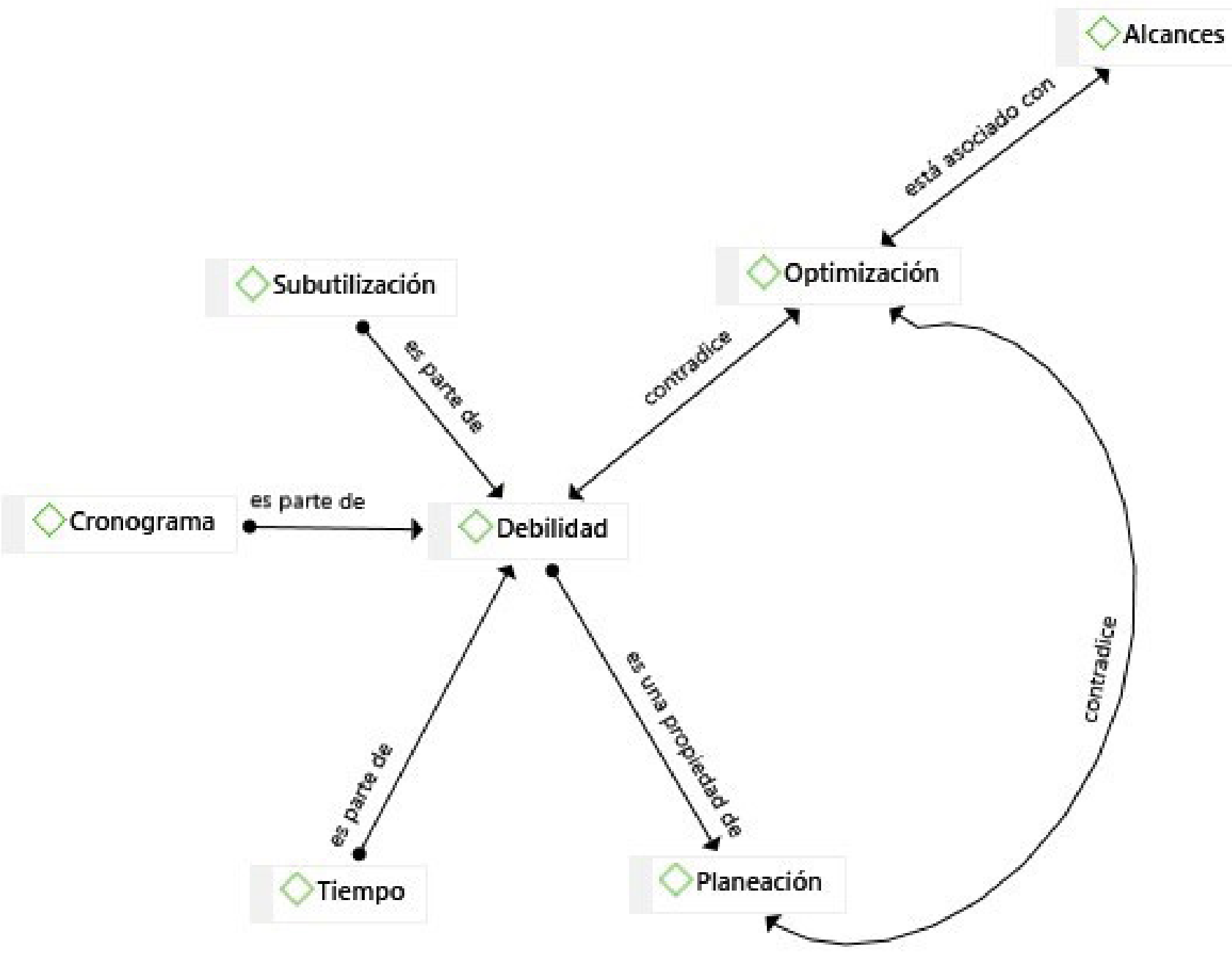

Figura 4. Familia Planeación

Fuente: Elaboración propia a partir de las entrevistas. 
Estatus de la organización en la gestión de proyectos, según el modelo de Harold Kerzner

Para la tipificación de la organización dentro del modelo de Harold Kerzner, se realizó la siguiente matriz (Tabla 4) de comparación de resultados, construida a partir de las coincidencias encontradas entre la fase cualitativa y cuantitativa de la organización, así:

TABLA 4.

Triangulación de Hallazgos

\begin{tabular}{|c|c|}
\hline Componente Cuantitativo & Componente Cualitativo \\
\hline \multicolumn{2}{|c|}{ Gestión Económico-Financiera de los Proyectos. } \\
\hline $\begin{array}{l}\text { En las encuestas se muestra una calificación } \\
\text { baja en torno a Costo y Riesgos, lo que } \\
\text { compromete a la gestión de los recursos en la } \\
\text { Organización, y, por ende, el éxito del proyecto. }\end{array}$ & $\begin{array}{l}\text { En las entrevistas se evidenció la preocupación } \\
\text { en torno al manejo de los recursos, tanto en los } \\
\text { momentos de austeridad como de bonanza, lo que } \\
\text { da cuenta de una debilidad sentida al interior de } \\
\text { la organización. }\end{array}$ \\
\hline \multicolumn{2}{|c|}{ Gestión Organizacional para los Proyectos } \\
\hline $\begin{array}{l}\text { Las encuestan dan cuenta de debilidades en } \\
\text { áreas estratégicas como los Recursos Humanos } \\
\text { y las Comunicaciones. Así mismo, muestran } \\
\text { un Logro de Compromisos calificado por debajo } \\
\text { del } 60 \% \text {, lo que es un factor critico para la } \\
\text { organización. }\end{array}$ & $\begin{array}{l}\text { Las entrevistan dan cuenta de debilidades en } \\
\text { torno al manejo de roles y de las estructuras de } \\
\text { poder, así mismo de un limitado aprovechamiento } \\
\text { de las lecciones aprendidas, lo que constituye una } \\
\text { debilidad para la consolidación de una cultura de } \\
\text { proyectos en la organización. }\end{array}$ \\
\hline \multicolumn{2}{|c|}{ Planeación y Desarrollo de los Proyectos } \\
\hline $\begin{array}{l}\text { Las encuestas permitieron evidenciar } \\
\text { debilidades en torno al tiempo - cumplimiento } \\
\text { del cronograma-, y el logro de compromisos, } \\
\text { lo que da cuenta de que la organización posee } \\
\text { limitantes en los procesos de planeación y } \\
\text { control de sus hitos. }\end{array}$ & $\begin{array}{l}\text { En las entrevistas afloró la preocupación latente } \\
\text { de los Directivos por las dificultades para el } \\
\text { cumplimiento de los cronogramas de los Proyectos, } \\
\text { considerando este aspecto como una debilidad } \\
\text { para el tejido administrativo de la compañía. }\end{array}$ \\
\hline
\end{tabular}

Fuente: Elaboración propia.

En virtud de los hallazgos descritos anteriormente en la Tabla 3, resulta dable afirmar que la organización presenta grandes oportunidades de mejora para la consolidación de una cultura de proyectos que garantice el éxito de los mismos. De ahí que, al observar los escaños definidos por Kerzner, la organización se encuentra en el primer nivel, es decir, lenguaje común, en el cual se "reconoce la necesidad de contar con un lenguaje único para comunicarse internamente con respecto a la forma como se administran sus proyectos" (Castellanos, Gallego, Delgado y Merchán, 2014). Al respecto, Cuero \& Pérez (2020), han expresado:

Dicha clasificación - por estar en el primer nivel - no es sinónimo de que la organización esté en precarias condiciones o que su pertinencia sea cuestionable, pues existen, como se describió con anterioridad, una serie de factores que sustentan su misionalidad. Por el contrario, es una oportunidad para implementar un proceso de (re)ingeniería organizacional que permita consolidar su quehacer dentro de la organización y asegurar el éxito integral de su cometido (p. 3). 


\section{Conclusiones}

Alcanzar un nivel adecuado de madurez en la gestión de proyectos, además de permitir el éxito de los mismos, le brinda a la organización una serie de beneficios adicionales que se traducen en procesos efectivos, por tanto, la consolidación de una cultura organizacional en torno a los proyectos debe ser una prioridad para los líderes y directivos, especialmente en entidades como la analizada en la presente investigación, pues dada su misionalidad (servicios públicos), además de una relación endógena (proyecto-empresa), existe un impacto directo de sus acciones sobre la calidad de vida en el territorio.

Respecto a los resultados específicos de los instrumentos, tanto las encuestas como las entrevistas, permitieron evidenciar que existe una incipiente cultura de proyecto dentro de la organización, situación que se evidencia al analizar las debilidades existentes en áreas estratégicas como el tiempo, alcance, costo, riesgos, comunicaciones, entre otros, lo que se traduce en una organización que debe replantear sus practicas e iniciar por reconocer la necesidad de establecer un lenguaje común, primer peldaño del modelo de Harold Kerzner, para la consolidación de una efectiva gestión de proyectos.

Resulta imperioso para la organización, tal vez como el primer paso para el fortalecimiento de su cultura de proyectos, la creación de un repositorio documental (banco de lecciones aprendidas), pues, dada la especificidad de su misionalidad, la mayoría de sus proyectos poseen rasgos comunes, y dicha herramienta resulta estratégica para la reducción de falencias y el mejoramiento de procesos. Igualmente, como parte de futuras investigaciones y/o intervenciones, resulta procedente explorar la efectividad de concentrar los proyectos en una dependencia especializada (PMO).

\section{ReCONOCIMientos y Agradecimientos}

El presente manuscrito es fruto del proyecto de investigación denominado "Evaluación del nivel de madurez para la gestión de proyectos en una empresa prestadora de servicios públicos en el departamento del Caquetá”.

\section{REFERENCIAS}

Arce, S. y López, H. (2010). Valoración de la gestión de proyectos en empresas de Bogotá. Nivel de madurez en gestión de proyectos. Revista EAN, (69), 60-87. https://doi.org/10.21158/01208160.n69.2010.517

Atlas ti. (versión 6). Análisis cualitativo de Datos. [Software]. Berlin: Scientific Software Development GmbH. Disponible en https://atlasti.com/es/

Castellanos, T., Gallego, J., Delgado, J. y Merchán, L. (2014). Análisis comparativo entre los modelos de madurez reconocidos en la gestión de proyectos. [Tesis de Especialización]. Universidad San Buenaventura de Cali, Santiago de Cali, Colombia. Disponible en http://bibliotecadigital.usb.edu.co/bitstre am/10819/2163/2/1131056_1131054_1131185_ANEXO_Cap\%C3\%ADtulo.pdf 
Cuero, E. y Pérez, M. (2020). Modelo para la implementación de la Oficina de Gestión de Proyectos (PMO), para la Oficina de Ciencia, Tecnología e Innovación del departamento del Huila. [Tesis de Maestría]. Universidad Externado de Colombia, Bogotá, Colombia. Disponible en https://bdigital.uexternado.edu.co/ handle/001/3566

Del Cioppo, J. y Bello, M. (2018). Indicadores de impacto social para evaluación de proyectos de vinculación con la colectividad. Económicas CUC, 39(1), 105-116. http://dx.doi.org/10.17981/econcuc.39.1.2018.07

Gómez, C. (2019). Diseño de una propuesta de implementación de una PMO (Project Management Office) en el Comité Departamental de Ganaderos del Caquetá. [Tesis de Maestría]. Universidad Externado de Colombia, Bogotá, Colombia. Disponible enhttps://bdigital.uexternado.edu.co/handle/001/2540

Gómez, C., Sánchez, V. y Fajardo, M. (2018). Los proyectos y sus dimensiones: una aproximación conceptual. Contexto, 7, 57-64. Disponible en https://revistas. ugca.edu.co/index.php/contexto/article/view/885

Gómez, C., Sánchez, V. y Ramón, L. (2017). Incorporar las TIC a los procesos de enseñanza-aprendizaje: Una lectura desde el actuar docente en educación superior. Horizontes Pedagógicos, 19(1), 48-54. https://doi.org/10.33881/0123-8264. hop.19106

Gómez, R. (2009). Manual de Gestión de Proyectos. Medellín: Editorial Universidad de Antioquia. Recuperado de https://abacoenred.com/wp-content/uploads/2015/10/ manual_gestion_proyectos.pdf

Hernández, R., Fernández, C. y Baptista, L. (2014). Metodología de la Investigación. 6 ed. México, D.F.: Mc Graw Hill.

Kerzner, H. (2005). Using the Project Management Maturity Model. (2 Ed.). USA: Jhon Wiley \& Sons.

Moreno, N., Sánchez, L. y Velosa, J. (2018). Introducción a la gerencia de proyectos: conceptos y aplicación. Bogotá, D.C.: Ediciones EAN. Disponible en http://hdl. handle.net/10882/9547

Ñungo, L., Torres, B. y Palacios, J. (2018). Modelo de nivel de madurez para los procesos de emprendimiento en las pymes colombianas. Revista Ingeniería Solidaria, 14(26), 1-17. https://doi.org/10.16925/in.v14i26.2456

Parviz, F. \& Levin, G. (2002). The advanced Project Management Office. Boca Raton: St. Lucie Press. https://doi.org/10.1201/9781420000375

PMI. (mar. 5, 2021). What is Project Management? pmi.org. Available: https://www. pmi.org/about/learn-about-pmi/what-is-project-management

PMI. (2017). Guía de los Fundamentos para la Dirección de Proyectos - Guía PMBOK ( 6 ed.). México, D.F.: South- Western.

Shek, I. (2013). El enfoque de gestión de proyectos en las organizaciones dedicadas a proyectos de investigación. Caso: Grupo de Investigación GIRH. Revista EAN, (74), 152-161. Disponible en https://journal.universidadean.edu.co/index.php/ Revista/article/view/742 
Sparano, H. (2011). Impacto de las áreas de conocimiento de la administración de proyectos a través del PMBOK 2008. Dimensión Empresarial, 9(1), 64-73. Disponible en http://ojs.uac.edu.co/index.php/dimension-empresarial/index

Teherán, Y. y Torres, J. (julio, 2019). Diseño de un modelo de evaluación y medición de la madurez organizacional en gestión de proyectos y aplicación a compañías de armadores de Colombia. Conferencia presentada 17th LACCEI International Multi-Conference for Engineering, Education, and Technology, LACCEI, Montego Bay, Jamaica. https://doi.org/10.18687/LACCEI2019.1.1.385

Terrazas, R. (2009). Modelo conceptual para la gestión de proyectos. Perspectivas, (24), 165-188. Disponible en https://www.redalyc.org/articulo.oa?id=425942160009

Triana, S. (2018). Modelo para el diagnóstico de madurez en la gestión de proyectos para MIPYMES de mantenimiento del sector metalmecánico en la Localidad de Kennedy. [Tesis de Maestría]. Universidad Militar Nueva Granada, Bogotá, D.C., Colombia. Recuperado de http://hdl.handle.net/10654/18140

Wendler, R. (2012). The maturity of maturity model research: A systematic mapping study. Information and Software Technology, 54(2), 1317-1339. https:// doi.org/10.1016/j.infsof.2012.07.007

BIODATA

Carlos Alberto Gómez Cano es Contador Público de la Universidad de la Amazonia (Colombia). Especialista en Gestión Pública de la Escuela Superior de Administración Pública-ESAP (Colombia). Magister en Gestión y Evaluación de Proyectos de Inversión de la Universidad Externado de Colombia. Docente-Investigador de la Corporación Unificada Nacional de Educación Superior-CUN (Colombia). ORCID: https://orcid.org/0000-0003-0425-7201

Verenice Sánchez Castillo es Ingeniera Agroecologa de la Universidad de la Amazonia (Colombia). Magister en Ambiente y Desarrollo de la Universidad de Puebla (Mpexico). Doctora en Antropología de la Universidad del Cauca (Colombia). Docente-Investigadora de la Facultad de Ingeniería de la Universidad de la Amazonia. ORCID: https://orcid.org/0000-0002-4472-6191 